\title{
Vestibular involvement in adults with HIVIAIDS
}

\author{
Barbara M. Heinze ${ }^{a^{*}}$, Bart M. Vinck ${ }^{a, b, 1}$, Louis M. Hofmeyr ${ }^{c, 2}$, De Wet Swanepoel ${ }^{a, d, e, 1}$ \\ ${ }^{\mathrm{a}}$ Department of Speech-Language Pathology and Audiology, University of Pretoria, Pretoria, South Africa \\ ${ }^{\mathrm{b}}$ Department of Audiology, University of Gent, Gent, Belgium \\ ${ }^{\mathrm{c}}$ Department of Otorhinolaryngology, University of Pretoria, Pretoria, South Africa \\ ${ }^{\mathrm{d}}$ Ear Sciences Centre, School of Surgery, University of Western Australia, Nedlands, Australia \\ ${ }^{\mathrm{e}}$ Ear Science Institute Australia, Subiaco, Australia
}

\begin{abstract}
* Corresponding author at: Department of Speech-language Pathology and Audiology, Private Bag X20, Hatfield, Pretoria 0028, South Africa. Tel.: +27 12 4205358; fax: +27 12 4203517. E-mail addresses: barbara.heinze@up.ac.za (B.M. Heinze), bart.vinck@up.ac.za (B.M. Vinck), Imhofmeyr@surgeon.co.za (L.M. Hofmeyr), dewet.swanepoel@up.ac.za (D.W. Swanepoel).

${ }^{1}$ Address: Department of Speech-Language Pathology and Audiology, Private Bag X20, Hatfield, Pretoria 0028, South Africa.

${ }^{2}$ Address: Department of Otorhinolaryngology, Private Bag X20, Hatfield, Pretoria 0028, South Africa.
\end{abstract}

\section{Abstract}

\section{Objective}

HIVIAIDS is responsible for widespread clinical manifestations involving the head, and neck. The prevalence and nature of vestibular involvement is still largely unknown. This study, aimed to describe and compare the occurrence and nature of vestibular involvement among a group of, adults infected with HIV compared to a control group. It also aimed to compare the vestibular function, of symptomatic and asymptomatic HIV positive adults who receive antiretroviral (ARV) therapies to, subjects not receiving ARV.

\section{Methods}

A cross-sectional study was conducted on 53 adults (29 male, 24 female, aged 23-49 years, mean $=38.5, \mathrm{SD}=4.4)$ infected with HIV, compared to a control group of $38 \mathrm{HIV}$ negative adults $(18$, male, 20 female, aged $20-49$ years, mean $=36.9, S D=8.2)$. $A$ structured interview probed the subjective, perception of vestibular symptoms. Medical records were reviewed for CD4+ cell counts and the use of, ARV medication. An otologic assessment and a comprehensive vestibular assessment (bedside, assessments, vestibular evoked myogenic potentials, ocular motor and positional tests and bithermal, caloric irrigation) were conducted. 


\section{Results}

Vestibular involvement occurred in $79.2 \%$ of subjects with HIV in all categories of disease, progression, compared to $18.4 \%$ in those without HIV. Vestibular involvement increased from $18.9 \%$ in CDC category 1 to $30.2 \%$ in category 2 . Vestibular involvement was $30.1 \%$ in category 3 . There were, vestibular involvement in $35.9 \%$ of symptomatic HIV positive subjects, and $41.5 \%$ in asymptomatic, HIV positive subjects. There was no significant difference in the occurrence of vestibular involvement, in subjects receiving ARV therapies compared to those not receiving ARV therapies ( $p=.914$; chi-square, test). The odds ratio indicates that individuals with HIV have a 16.61 times higher risk of developing, vestibular involvement during their lifetime of living with the disease and that it may occur despite, being asymptomatic.

\section{Conclusion}

Vestibular involvement was significantly more common in subjects with HIV. Primary health care providers could screen HIV positive patients to ascertain if there are symptoms of vestibular involvement. If there are any, then they may consider further vestibular assessments and subsequent vestibular rehabilitation therapy.

\section{Keywords}

- Human immunodeficiency virus (HIV);

- Acquired immunodeficiency syndrome (AIDS);

- Vestibular involvement;

- Vertigo;

- CD4+ cell count;

- Antiretroviral (ARV) therapies

\section{Introduction}

The human immunodeficiency virus (HIV) and acquired immunodeficiency syndrome duration (AIDS) is a world-wide pandemic affecting the lives of millions of people. Despite the number of new infections and AIDS-related deaths decreasing between 2001 and 2009, the overall number of individuals living with the disease is still very high [1]. Increased life expectancy and of survival of those living with HIVIAIDS may, among other factors such as public health education and awareness programmes, be attributed to antiretroviral (ARV) therapy and improvements in access to health care services. The reduction in morbidity and mortality is changing HIVIAIDS from a life-threatening disease to a chronic illness with an increasing emphasis on quality of life issues [2].

After HIV binds with and penetrates into the CD4+ cell, it compromises the body's immune response gradually and predisposes it to opportunistic infections [3,4]. Diseases involving 
the head and neck area are often the first signs of an immune compromised body and may occur in as many as $40 \%$ to $90 \%$ of individuals with HIVIAIDS [5-9]. Common HIVIAIDSassociated head and neck pathologies include manifestations in the central nervous system (CNS), the naso-, oro- and laryngopharynx and in the inner ear, that may result in hearing loss and/or vestibular disorders [10-12].

There is growing evidence that HIVIAIDS could either directly or indirectly, through opportunistic infections or ototoxic medication, have an adverse effect on the delicate structures of the ear, causing a conductive, mixed or sensorineural hearing loss [13-19]. Auditory dysfunction has been reported to occur in between $20 \%$ to $75 \%$ of adults with HIVIAIDS [20-23]. The auditory and vestibular system is situated in the temporal bone and constitutes part of the same membranous labyrinth. It is not surprising that with auditory symptoms, which often accompany HIVIAIDS, vestibular dysfunction and associated symptoms may also occur. Vestibular symptoms may include dizziness, vertigo, disequilibrium and/or nausea and vomiting.

However, only a limited number of studies have investigated vestibular dysfunction and pathology related to HIVIAIDS, despite the shared anatomy and physiology of the auditory system [2]. Teggi and colleagues [18] suggested that vestibular symptoms are often masked by other more serious and life-threatening illnesses and disorders associated with HIVIAIDS. As a result, the nature of vestibular symptoms and the potential for manifestations of vestibular pathology resulting from HIVIAIDS have been largely neglected.

The most recent and extensive reports were group studies of adults [17,18,24,25] and children [16] infected with HIV who underwent auditory and vestibular assessments. The findings demonstrated vestibular dysfunction to be more frequent among subjects with HIV than those without HIV. Signs of both peripheral (involving the vestibular end-organs and eighth cranial nerve) and central (vestibular nuclei in the brainstem, cerebellum and oculomotor, vestibulospinal and proprioceptive pathways) vestibular dysfunction have been reported [16-18,24,25]. Vestibular dysfunction occurred across all categories of the disease with a higher prevalence in more advanced categories, particularly regarding central vestibular involvement $[17,18,26]$. These studies employed various tests of 
vestibular function, included spontaneous and positional/positioning nystagmus tests, ocular motor tests, caloric tests and posturography. Cervical vestibular evoked myogenic potentials (cVEMPs) have recently been introduced in the clinical evaluation of the saccule (vestibular end-organ) and inferior vestibular nerve. However, no study has yet utilized cVEMPs as part of a vestibular test battery in individuals with HIV to determine peripheral involvement.

One study compared the vestibular functioning of 60 HIV positive adults suffering from vestibular symptoms with 30 HIV negative adults also with vestibular symptoms [18]. This study showed a higher number of abnormal peripheral and central vestibular findings among the HIV positive group than among the HIV negative group. Another study compared vestibular functioning of HIV positive and HIV negative adults without any vestibular symptoms [24] and reported central vestibular involvement in the HIV positive group. No signs of peripheral vestibular involvement were however reported in the sample of asymptomatic subjects. Both of these studies utilised essentially the same test procedures, namely spontaneous and positional nystagmus tests, ocular motor tests and caloric tests, with the exception of the auditory brainstem response included in the latter. It is therefore not clear if HIV affects the peripheral vestibular system in asymptomatic individuals. It is also not clear if the vestibular function of symptomatic HIV positive individuals would differ from asymptomatic HIV positive individuals. No studies to date have compared the peripheral and central vestibular functioning of HIV positive individuals with and without vestibular symptoms.

There are also no studies that described peripheral and central vestibular functioning in adults with HIV receiving ARV therapies compared to those without this treatment. The current study aimed (i) to describe the occurrence and nature of vestibular involvement among a group of adults infected with HIV, compared to a control group without HIV by including cVEMPs in the vestibular test battery; (ii) to describe and compare the vestibular function of symptomatic and asymptomatic HIV positive adults; (iii) to describe and compare the vestibular function of a group of adults with HIV receiving ARV therapies to those not receiving ARV therapies. 


\section{Materials and methods}

\subsection{Ethical clearance and informed consent}

The institutional review boards of the University of Pretoria and the tertiary referral hospital where subjects were registered, reviewed and approved the study before any data collection commenced. Subjects provided written informed consent for the researcher to access their medical records, to have access to their HIV status, to document the CD4+ cell count and the use of ARV therapies.

\subsection{Study design}

A cross-sectional comparative research design was employed. A convenience sampling method was used to recruit subjects.

\subsection{Subjects}

Table 1 summarizes the description of participating subjects. A total of 91 subjects participated, comprising 53 HIV positive and 38 HIV negative adults. There were no statistically significant differences in mean ages between the groups ( $p=0.26$; t-test) and therefore there was not a difference in age groups. Since age only affects the vestibular system after 55 to 65 years [27], the age of the subjects were below 50 in order to minimize the likelihood of age affecting the results. Age distribution was the same across the two study groups (Mann-Whitney $U$ test). In addition, gender distribution was similar between and within the groups. HIV positive subjects were evenly distributed between the three Centre for Disease Control and Prevention (CDC) classification categories $(p>0.05$; one-way ANOVA). Fifteen subjects were in category 1 (eight male, seven female), 20 subjects were in category 2 (eight male, 12 female) and 18 subjects were in category 3 (eight male, 10 female). Table 1 furthermore shows the ARV therapy regimes for the subjects with HIV. There were 42 subjects with HIV who used ARV therapies and 11 subjects with HIV who did not use ARV therapies at the time of entry in the study. 
Table 1

Description of subjects

\begin{tabular}{lll}
\hline Description & HIV negative group & HIV positive group \\
\hline Number of subjects $(\mathbf{n})$ & 38 & 53 \\
Mean age & $36.9(\mathrm{SD}=8.2)$ & $38.5(\mathrm{SD}=4.4)$ \\
Min-Max age & $20-50$ years & $23-49$ years \\
Gender distribution & Male $=47.4 \%(n=18)$ & Male $=55 \%(n=29)$ \\
& Female $=52.6 \%(n=20)$ & Female $=45 \%(n=24)$ \\
CDC categories & Not applicable & CDC category $1: n=15$ \\
& & CDC category $2: n=20$ \\
& & CDC category $3: n=18$ \\
ARV therapies & TDF+ FTC+NVP: $n=11$ \\
& Not applicable & d4T+3TC+NVP: $n=4$ \\
& & TDF+3TC+EFV: $n=13$ \\
& TDF+3TC+LPV/r: $n=3$ \\
& AZT+3TC+EFV: $n=2$ \\
& AZT+3TC+LPV/r: $n=1$ \\
& & Unknown/not documented: $n=8$ \\
& non-ARV therapy users: $n=11$
\end{tabular}

Abbreviations: SD, Standard Deviation; CDC, Centres for Disease Control; ARV, antiretroviral; TDF, tenofovir; 3TC, lamivudine; EFV, efavirenz; FTC, emtricitabine; NVP, nevirapine; d4T, stavudine; AZT, zidovudine; LPV/r, lopinavir/ritonavir.

\subsubsection{Experimental group}

Subjects in the experimental group were HIV positive patients from the Infectious Disease (ID) clinic of a tertiary referral hospital in South Africa. The majority of recruited patients were HIV positive. Those who were HIV negative $(n=1)$ were assigned to the control group.

The inclusion criteria were a positive diagnosis of HIV as determined by blood serological tests; aged between 18-50 years since this age group has the highest prevalence of HIVIAIDS [28] and to minimize age as a contributing influence on results; and any ethnic group and gender. The following conditions excluded participation by potential candidates: exposure to ototoxic agents prior to contracting HIVIAIDS; a history of vestibular involvement and/or complaints of dizziness, vertigo or disequilibrium prior to contracting HIVIAIDS, thus reducing the likelihood of other vestibular disorders affecting the results; blindness or other disorders of vision that would not allow Video Nystagmography (VNG). 
The subjects with HIV were further divided into categories according to their CD4+ cell counts at entry in the study. Subjects with counts higher than 500 cells/uL were assigned to CDC category 1 , while those with counts of $200-499$ cells/uL and less than 200 cells/uL were assigned to categories 2 and 3 respectively.

\subsubsection{Control group}

Subjects in the control group were patients from the ID clinic confirmed not to have HIV, acquaintances of the researcher and employees of the tertiary referral hospital. Individuals were required to agree to an HIV blood serological test before being included. Once the latter two groups agreed and gave written informed consent, an HIV test was conducted by medical staff at the tertiary referral hospital involved in this study. These test incurred no costs to subjects and the results were documented and managed by medical staff. The inclusion criteria were: HIV negative; aged between 18-50 years to account for normal aging of auditory and vestibular structures; and any ethnic group and gender. Exclusion criteria were reported previous exposure to ototoxic agents; and blindness or other disorders of vision.

\subsection{Procedures}

Table 2 summarizes the data collection protocol, its sequence and purpose. Prior to data collection, subjects were provided with a letter requesting of informed consent that also explained the procedures for the auditory and vestibular assessments as well as a written list of pre-test instructions. The list of pre-test instructions explained that subjects were not permitted to take medication that may suppress vestibular symptoms, or agents such as alcohol and/or sedatives that may suppress peripheral and/or central vestibular function and influence the results and their interpretation. The procedures for the first two points in Table 2 were a medical record review to document subjects' CD4+ cell counts at the time of participation in the study, use of antiretroviral agents and a structured interview. We used the questionnaire from Goebel [29] to probe for any subjective perception of vestibular symptoms. The remainder of the test procedures as indicated in Table 2 were performed in a single session lasting approximately two hours. 
Table 2

Summarised data collection protocol according to test sequence

Category and sequence

1. Medical record review

2. Structured interview

3. Otologic and audiologic examination

4. Evoked potentials

5. Vestibular clinical/bedside assessments

6. Ocular motor tests*

7. Positioning/positional tests*

8. Caloric irrigation*
Purpose / tests

Document CD4+ cell counts, use of antiretroviral therapies.

Subjective perception of any vestibular symptoms (questionnaire from Goebel [29]).

Otoscopy, tympanometry, pure tone audiometry

Cervical Vestibular Evoked Myogenic Potentials (cVEMP)

Fukuda stepping test, subjective visual vertical test, head impulse test, dynamic visual acuity, spontaneous nystagmus test ${ }^{*}$, head shake test ${ }^{*}$, hyperventilation test $^{*}$

Gaze evoked nystagmus testing (horizontal and vertical), random saccade testing, pursuit tracking

Dix-Hallpike, positional body, positional head

Bithermal caloric irrigation, directional preponderance

Note: *Video nystagmography (VNG) with binocular infrared goggles were used to observe and record eye movements and nystagmus

\subsubsection{Otologic and audiological examination}

Outer ear functioning was assessed through an otoscopic examination. A physician managed the ear canals of subjects with impacted cerumen prior to the vestibular evaluation. Tympanometry was performed using a diagnostic Y-226 Hz probe tone (GSI Tympstar, Grason-Stadler, Eden Prairie, MN, USA). The following criteria [30] were used for normal adult admittance profiling: ear canal volume $(0.8$ to $2.0 \mathrm{ml})$, compliance (0.3 to $1.8 \mathrm{ml}$ ) and middle ear pressure (-100 daPa to $+50 \mathrm{daPa}$ ). Pure tone audiometry (air and bone conduction) was performed to determine the presence of air-bone gaps. VEMP data was not analysed if air-bone gaps were greater than $10 \mathrm{~dB}$. 


\subsubsection{VEMP testing}

cVEMPs were measured with the non-inverting electrode placed on the medial portion of the sternocleidomastoid muscle (SCM), the inverting electrode on Fpz and ground on $\mathrm{Fz}$ (Biologic Navigator Pro, Natus Medical Incorporated, San Carlos, CA, USA). Skin impedance was less than $5 \mathrm{k} \Omega$. Wester [31] described this electrode montage. Colebatch and colleagues [32] as well as Vanspauwen and colleagues [33] described a similar electrode montage, with the exception of the inverting electrode on the upper sternum. In this study, cVEMPs from 24 normal ears without a history of vestibular dysfunction with the inverting electrode on Fpz (montage 1) were recorded and compared with the inverting electrode on the upper sternum (montage 2$)$. The mean amplitude was $146.57( \pm 44.75)$ and $158.31( \pm 53.88)$ for montage 1 and 2 respectively, which was statistically insignificant $(p=.098 ;$ t-test). The mean P1 latency was $13.478( \pm 0.84)$ and $13.63( \pm 0.95)$ for montage 1 and 2 respectively, which was statistically insignificant ( $p=.34$; t-test). The mean N1 latencies were $21.875( \pm 1.34)$ and $21.815( \pm 1.42)$ for montage 1 and 2 respectively, which was statistically insignificant ( $p=.824$; t-test). Alternating polarity tone burst $(750 \mathrm{~Hz})$ stimuli were presented at $95 \mathrm{dBnHL}$ monaurally with insert earphones. A blood pressure manometer with rolled up inflatable cuff was positioned between the subjects' hand and jaw as feedback method of the contracted SCM muscle during recordings. A sustained pressure of $40 \mathrm{mmHg}$ was obtained to ensure comparable muscle contraction between both sides. This method was described by Vanspauwen and colleagues [33] as well as by Maes and colleagues [34] and was proved to provide reliable VEMP amplitudes. All measurements, both present and absent responses, were repeated to test for wave repeatability. The first positive peak on the waveforms was marked $\mathrm{P} 1$, while the first negative deflection was marked N1. The following VEMP asymmetry ratio formula was used [35]: $\left[\left(A_{L}-A_{R}\right) /\left(A_{L}+A_{R}\right)\right] \times 100$. ' $A_{L}$ ' indicated the amplitude for the left ear and ' $A_{R}$ ' the amplitude for the right ear. Responses were interpreted as follows: (i) the absence of unilateral or bilateral waveforms were considered abnormal (absence of an identifiable P1 and N1), (ii) two standard deviations above the mean of the HIV negative group were used to calculate the upper limits for $\mathrm{P} 1$ and $\mathrm{N} 1$ latencies (17.0ms and $26.3 \mathrm{~ms}$ respectively). Latencies above these upper limits were regarded as present yet delayed, and considered abnormal and (iii) the presence of an amplitude asymmetry ratio of $\geq 40 \%$ was considered abnormal, since it indicates side-to-side amplitude differences [35]. 


\subsubsection{Vestibular clinical/bedside tests}

Vestibular clinical/bedside tests that were conducted are listed in Table 2. During the Fukuda stepping test, subjects were asked to step or march 50 times with eyes closed and arms extended. A sideward rotation greater than 45 degrees were considered pathological. For the subjective visual vertical (SVV) test the the bucket method was used, described and illustrated elsewhere [36], as bedside tool to detect abnormal subjective tilt. The subjects looked into the bucket without having gravitational orientation clues and had to vertically straighten a line on the bottom inside of the bucket. The average of five repetitions was taken. Offsets greater than three degrees were considered pathological. During the horizontal head impulse test the investigator stood in front of the subject and firmly held the head while rapidly rotating the head to both the left and right side by $20-30^{\circ}$. The subjects had to maintain visual fixation and corrective eye movements; failure of visual fixation during these rapid head movements were considered pathological. Dynamic visual acuity testing was performed using a computerized VNG/ENG system (Micromedical Technologies Inc., Chatham, IL, USA). A visual optotype appeared when the head velocity exceeded 100 degrees per second and blanked during periods of low head velocity. The head was oscillated horizontally and vertically at a frequency of $2 \mathrm{~Hz}$. Subjects had to identify the direction of the visual optotype correctly. Incorrect identification of the visual optotype that increased 2 or more times from baseline visual acuity during head rotation were considered pathological.

Next, the four channel Visual Eyes infrared video-based system (Micromedical Technologies Inc., Chatham, IL, USA) was used to record eye movements. The presence of spontaneous nystagmus was evaluated with and without fixation, and such presence was considered pathological. Subsequently, the subject's head was moved sinusoidally in the horizontal (yaw) plane at a cycle of $2 \mathrm{~Hz}$ for $25-30$ seconds and the presence of post headshake induced nystagmus was noted with vision denied. Post head shake nystagmus was considered pathological. Subjects were then asked to deeply inhale and exhale at a cycle of $1 \mathrm{~Hz}$ for 45 seconds during which nystagmus induced by hyperventilation was noted with vision denied. The presence of nystagmus induced by hyperventilation was considered pathological. 


\subsubsection{Ocular motor tests}

For the ocular motor tests the same infrared video goggles were used, during which eye movements were recorded. A digital light bar was used to perform the ocular motor tests. Age and visual acuity may affect the outcomes of the ocular motor tests $[37,38]$, therefore age was accounted for when selecting subjects, as well as their visual acuity. Gaze evoked nystagmus evaluated the subject's abilities to maintain gaze in eccentric positions, both horizontally and vertically. The presence of gaze evoked nystagmus was considered pathological. Random saccade testing was analysed according to the following parameters: velocity, latency and accuracy of the eye movements. Pursuit tracking was analysed for smooth eye movements, according to the following parameters: gain, symmetry and phase at the velocities of $0.1 \mathrm{~Hz}, 0.2 \mathrm{~Hz}$ and $0.4 \mathrm{~Hz}$. Any abnormalities in saccades and pursuit were considered pathological. Optokinetic testing was not performed because a full visual field stimulus was unavailable at the time.

\subsubsection{Positioning/positional tests}

Dix-Hallpike testing and various positional head and body nystagmus tests (also with vision denied) were performed using the infrared video goggles. The presence of nystagmus induced by position was considered pathological.

\subsubsection{Caloric irrigation}

Finally, a bithermal, binaural air caloric test (AirFX, Micromedical Technologies Inc., Chatham, IL, USA) was performed to evaluate individual horizontal semi-circular canal responsiveness. This system allowed visualization of the entire ear canal and tympanic membrane, and was safe to use for subjects with outer and/or middle ear infections and/or tympanic membrane perforations, without risking further infection in comparison to water caloric tests. The air currents were 47 and 24 degrees Celsius respectively and the stimulus was applied for 60 seconds, with an interval of five minutes between irrigation. Jongkees' formula [39] was used to calculate unilateral weakness or asymmetry and directional preponderance: $(R W+R C)-(L W+L C) /(R W+R C+L W+L C) \times 100 . A$ unilateral weakness or asymmetry of $\geq 20 \%$, directional preponderance of $\geq 30 \%$, bilateral weakness and hyperreflexia (total response more than 221 degrees per second) was considered abnormal. 


\subsubsection{Identification of peripheral signs}

The presence of at least one of the following findings suggested signs of a peripheral vestibular involvement: (i) spontaneous nystagmus inhibited by fixation and fixed direction; (ii) re-fixation or catch-up saccadic eye movement with the head impulse test; (iii) horizontal post head shake nystagmus; (iv) rotation greater than 45 degrees with the Fukuda stepping test; (v) vertical offsets $\geq 3$ degrees without concomitant skew deviation and/or ocular tilt; (vi) horizontal nystagmus induced by hyperventilation ; (vii) abnormal dynamic visual acuity; (viii) nystagmus evoked by direction-fixed gaze that follows Alexander's law and inhibited by fixation; (ix) position induced torsional upbeat, horizontal or torsional downbeat nystagmus with a latency of three to five seconds; a duration less than 60 seconds and fixation suppresses or inhibits nystagmus; $(x)$ caloric unilateral weakness of $20 \%$ or greater, bilateral weakness with a history of a vestibular disorder or exposure to ototoxic agents and fixation suppression response; (xi) absent cVEMP recordings, asymmetry ratio $\geq 40 \%$ and delayed latencies.

\subsubsection{Identification of central signs}

The presence of at least one of the following findings suggested signs of central vestibular involvement: (i) spontaneous nystagmus not inhibited by fixation, direction changing nystagmus; (ii) perverted (vertical or torsional) post head shake nystagmus; (iii) vertical offsets $\geq 3$ degrees with concomitant skew deviation and/or ocular tilt; (iv) vertical hyperventilation induced nystagmus; (v) direction changing nystagmus, vertical nystagmus and nystagmus enhanced with fixation; (vi) abnormalities in random saccade velocities, accuracies or latencies; (vii) saccadic, asymmetric or absent pursuit; (viii) position induced vertical nystagmus without a latency and duration longer than 60 seconds, persistent nystagmus while in position and fixation and that does not inhibit or suppress nystagmus; (ix) bilateral caloric weakness without a history of a vestibular disorder or middle ear pathology, failure of fixation suppression, hyperreflexia, directional preponderance of $30 \%$ or greater without a unilateral weakness.

\subsection{Data analysis}

All analyses of data were performed using the statistical software package SPSS for

Windows version 21. Mean, standard deviation $( \pm)$ and percentages were used to describe the data. One-way analysis of variance (ANOVA) was used to compare the distribution of 
HIV positive subjects between the three CDC categories. The One-Sample KolmogorovSmirnov Test was used to demonstrate normality of data. The independent samples t-test was used to compare mean values between the experimental and control groups. $P$ values $<0.05$ were accepted as statistically significant. Effect sizes were determined using Cramer's V; and odds ratios were calculated. The chi-square non-parametric test was used to compare the findings between the two study groups and the three CDC categories.

\section{Results}

There was a significantly higher occurrence of vestibular involvement in subjects with HIVIAIDS ( $p=.001$; chi-square). Peripheral and central vestibular involvement was measured in $79.2 \%$ of subjects with HIV compared to $18.4 \%$ of subjects without HIV (Fig. 1). The effect size was large (Cramer's $V$ value $=.602$ ), demonstrating a strong association between vestibular signs and HIV. This association between vestibular signs and HIV was further confirmed by the odds ratio. A ratio of 16.61 was obtained, showing a 16.61 times higher risk for developing vestibular signs in persons who are HIV positive.

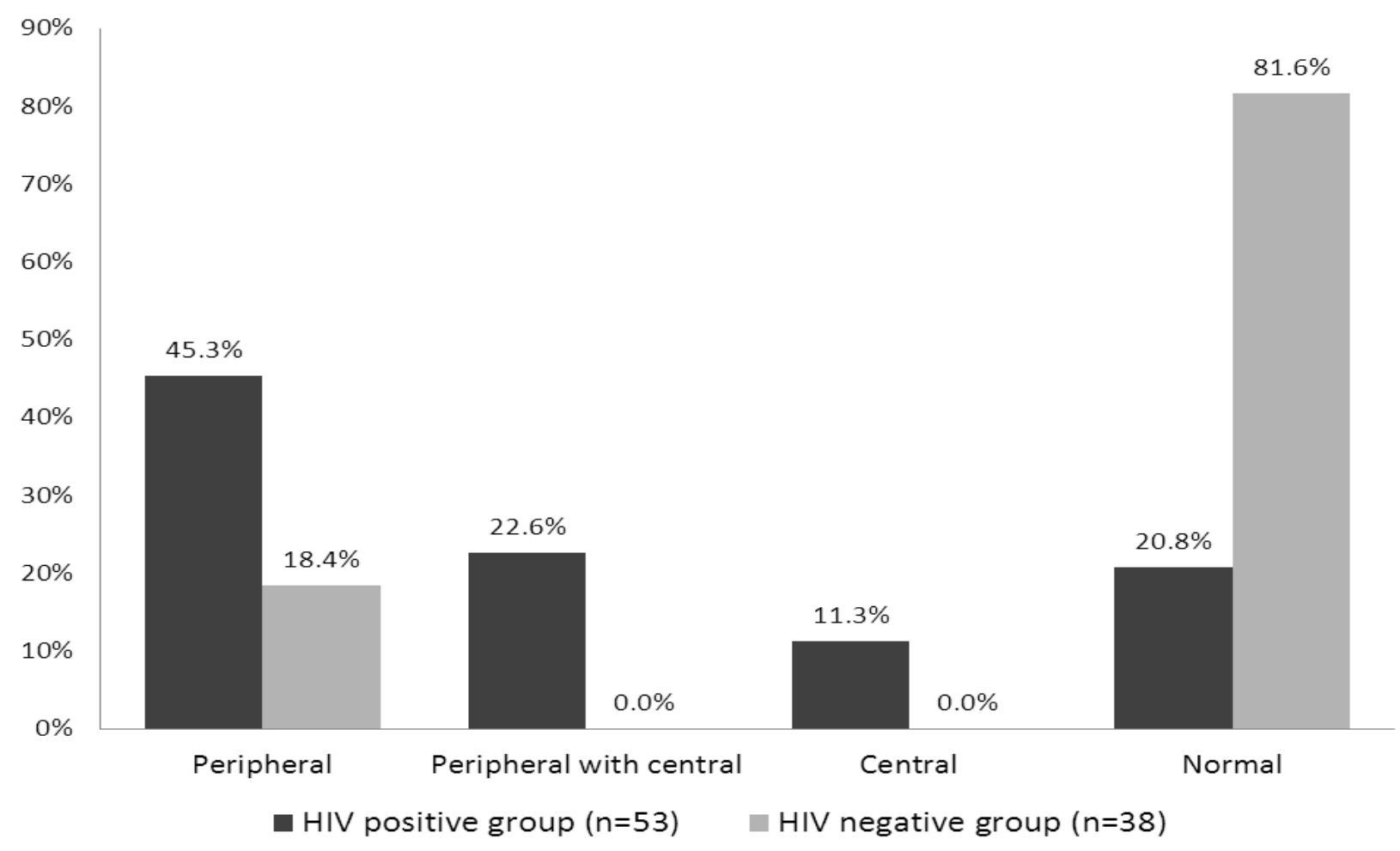

Fig. 1. Vestibular involvement across HIV positive and HIV negative groups 
Table 3

Distribution and number of abnormal vestibular tests results and its parameters in the HIV positive and negative groups

\begin{tabular}{|c|c|c|c|c|c|}
\hline \multirow{3}{*}{ Vestibular tests and parameters } & \multicolumn{4}{|c|}{ Subjects with abnormalities } & \multirow{3}{*}{$p$ value } \\
\hline & \multicolumn{2}{|c|}{ HIV positive group } & \multicolumn{2}{|c|}{ HIV negative group } & \\
\hline & $\mathbf{N}(\%)$ & mean (SD) & $\mathbf{N}(\%)$ & mean (SD) & \\
\hline \multicolumn{6}{|l|}{ Bithermal caloric tests } \\
\hline Unilateral weakness & $15(28.3)$ & $18.6(20.5)^{\mathrm{a}}$ & $3(7.9)$ & $10.1(16.4)^{a}$ & $.03^{d}$ \\
\hline Bilateral weakness ${ }^{\mathrm{e}}$ & $1(1.9)$ & - & $1(2.6)$ & - & $.81^{\mathrm{g}}$ \\
\hline Hyperreflexia $^{\dagger}$ & $2(3.8)$ & $233.5(13.4)$ & - & - & - \\
\hline DP & $3(5.7)$ & $35.3(4.0)$ & - & - & - \\
\hline \multicolumn{6}{|l|}{ cVEMP } \\
\hline Bilateral absent & $5(9.4)$ & - & $1(2.6)$ & - & _- \\
\hline Unilateral absent & $6(11.3)$ & - & $1(2.6)$ & - & - \\
\hline Asymmetry ratio & $10(18.9)^{\mathrm{b}}$ & $32.4(30.9)^{\mathrm{c}}$ & $3(7.9)^{b}$ & $15.3(17.2)^{\mathrm{c}}$ & $.002^{d}$ \\
\hline Delayed latencies & $9(17)$ & - & - & - & - \\
\hline \multicolumn{6}{|l|}{ Spontaneous nystagmus } \\
\hline Without fixation & $1(1.9)$ & - & - & - & - \\
\hline With fixation & - & - & - & - & - \\
\hline \multicolumn{6}{|l|}{ Head impulse test } \\
\hline Unilateral & $10(18.9)$ & & $3(7.9)$ & - & $.18^{\mathrm{g}}$ \\
\hline Bilateral & $1(1.9)$ & & $1(2.6)$ & - & $.81^{\mathrm{g}}$ \\
\hline \multicolumn{6}{|l|}{ Horizontal head shake test } \\
\hline Horizontal nystagmus & $13(24.5)$ & & $2(5.3)$ & - & $.03^{g}$ \\
\hline Vertical/torsional nystagmus & - & & - & - & - \\
\hline \multicolumn{6}{|l|}{ Fukuda stepping test } \\
\hline$\geq 45$ degrees deviation & $15(28.3)$ & & $2(5.3)$ & - & $.01^{\mathrm{g}}$ \\
\hline \multicolumn{6}{|l|}{ Subjective visual vertical test } \\
\hline$\geq 3$ degrees off vertical & $2(3.8)$ & & - & - & - \\
\hline \multicolumn{6}{|l|}{ Hyperventilation induced nystagmus } \\
\hline Horizontal nystagmus & $3(5.7)$ & & - & - & - \\
\hline Vertical nystagmus & - & & - & - & - \\
\hline \multicolumn{6}{|l|}{ Dynamic Visual Acuity } \\
\hline Unilateral & $2(3.8)$ & & - & - & - \\
\hline Bilateral & $1(1.9)$ & & - & - & - \\
\hline \multicolumn{6}{|l|}{ Gaze evoked nystagmus } \\
\hline Horizontal gaze & - & & - & - & - \\
\hline Vertical gaze & - & & - & - & - \\
\hline \multicolumn{6}{|l|}{ Random saccades } \\
\hline Velocity & $2(3.8)$ & & - & - & - \\
\hline Accuracy & -1 & & - & - & - \\
\hline Latency & - & & - & - & - \\
\hline \multicolumn{6}{|l|}{ Pursuit tracking } \\
\hline Saccadic pursuit & $9(17)$ & & - & - & - \\
\hline Asymmetric pursuit & - & & - & - & - \\
\hline Absent pursuit & - & & - & - & - \\
\hline \multicolumn{6}{|l|}{ Positioning/Positional tests } \\
\hline Torsional upbeat nystagmus & $5(9.4)$ & & - & - & - \\
\hline Torsional downbeat nystagmus & - & & - & - & - \\
\hline Horizontal nystagmus & $8(15.1)$ & & - & - & - \\
\hline Vertical nystagmus & $4(7.5)$ & & - & - & - \\
\hline \multicolumn{6}{|c|}{ 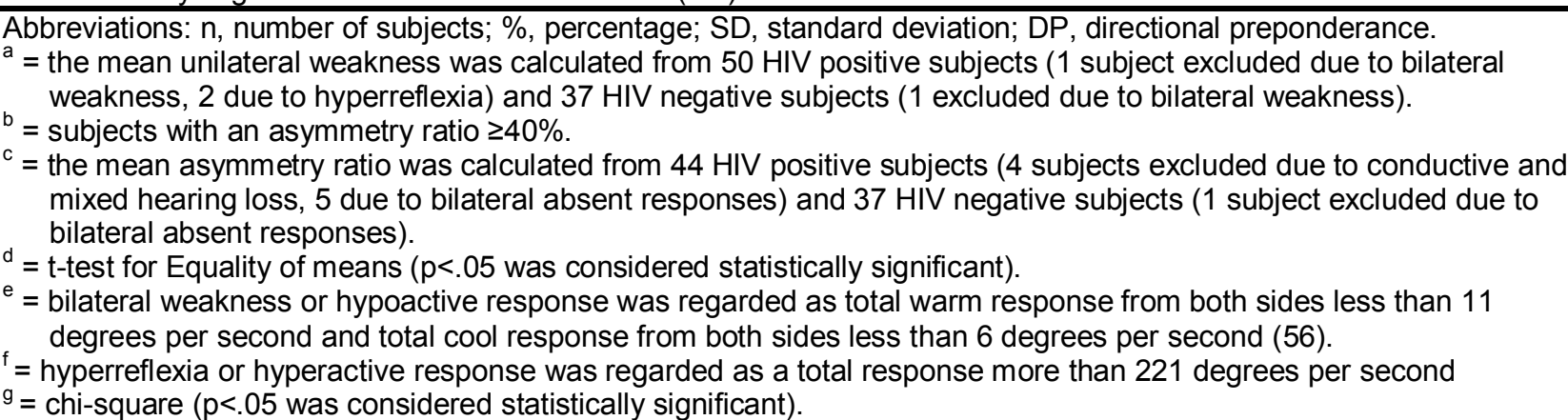 } \\
\hline
\end{tabular}


Almost half (45.3\%; $n=24)$ of the HIV positive subjects with vestibular involvement had signs of peripheral vestibular involvement, while $22.6 \%(n=12)$ had peripheral with central vestibular involvement and $11.3 \%(n=6)$ had central vestibular involvement (Fig. 1). Among the subjects without HIV, only peripheral vestibular involvement occurred.

The types of vestibular test abnormalities presented by the groups are included in Table 2 . Means were supplied for caloric and cVEMP tests due to the objective nature of the tests. Table 3 shows that the majority of caloric test abnormalities were due to unilateral weaknesses. In addition to caloric tests, the Fukuda stepping test revealed the most abnormalities. The horizontal head shake test and head impulse test revealed the second and third most abnormalities respectively. The HIV positive group demonstrated more abnormalities in different vestibular test procedures than the HIV negative group (13 versus five).

Vestibular involvement was measured across all CDC categories of HIV (Fig. 2). Vestibular involvement was $18.9 \%$ in CDC category $1,30.2 \%$ in category 2 and $30.1 \%$ in category 3 . There were no statistically significant differences in the occurrence of vestibular involvement between category 1 and 2 ( $p=.65$; chi-square) or between category 2 and 3 ( $p=.76$; chi-square). Figure 2 illustrates that there were peripheral vestibular involvement and both peripheral and central involvement in category 1 . Central signs (without peripheral signs) occurred in category 2 and category 3 , and although there was a noticeable increase in its occurrence from earlier to more advanced stages of disease progression (3.8\% to $7.5 \%)$, this was not statistically significant ( $p>.05$; chi-square). In addition, the occurrence of peripheral with central vestibular involvement increased with disease progression as well, but this was also not statistically significant $(p>.05$; chisquare). Peripheral vestibular involvement increased from category 1 to category 2 , but there was a slight decrease in its occurrence in category 3 , which was not statistically significant ( $p=.43$; chi-square). 


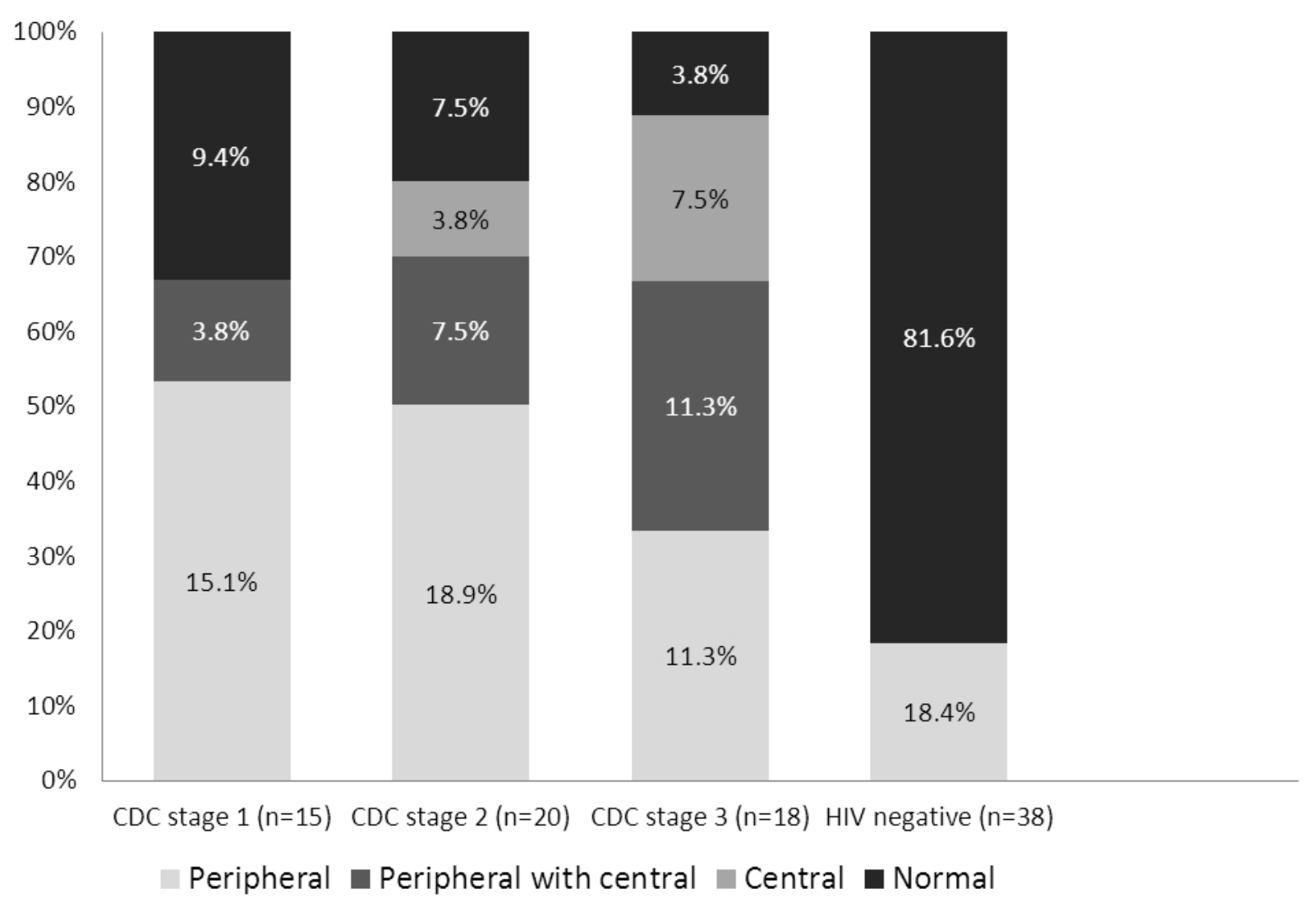

Fig. 2. Distribution of peripheral and central vestibular involvement across the CDC categories of HIV disease progression

Vestibular symptoms which included dizziness, vertigo, disequilibrium and/or nausea and vomiting were reported by $37.7 \%$ of HIV positive subjects $(n=20)$, while $62.3 \%(n=33)$ had no self-reported symptoms. In category 1 , six subjects were symptomatic and nine were asymptomatic. In category 2, nine subjects were symptomatic and 11 were asymptomatic. In category 3 , five were symptomatic and 13 were asymptomatic. None of the HIV negative subjects reported having these symptoms. There was vestibular involvement in $35.9 \%(n=19)$ of symptomatic HIV positive subjects compared to only one symptomatic subject $(1.9 \%)$ who had normal vestibular function ( $p=.001$; chi-square). In addition, there was a statistically significant ( $p=.05$; chi-square) higher occurrence of vestibular involvement among asymptomatic HIV positive subjects (43.3\%; $n=23$ ) compared to normal vestibular function in $20.7 \%(n=11)$ of asymptomatic subjects. 
Figure 3 compares the occurrence of vestibular involvement in HIV subjects with and without ARV therapies. There was no statistically significant difference between the occurrence of vestibular involvement in these two groups ( $p>.05$; chi-square).

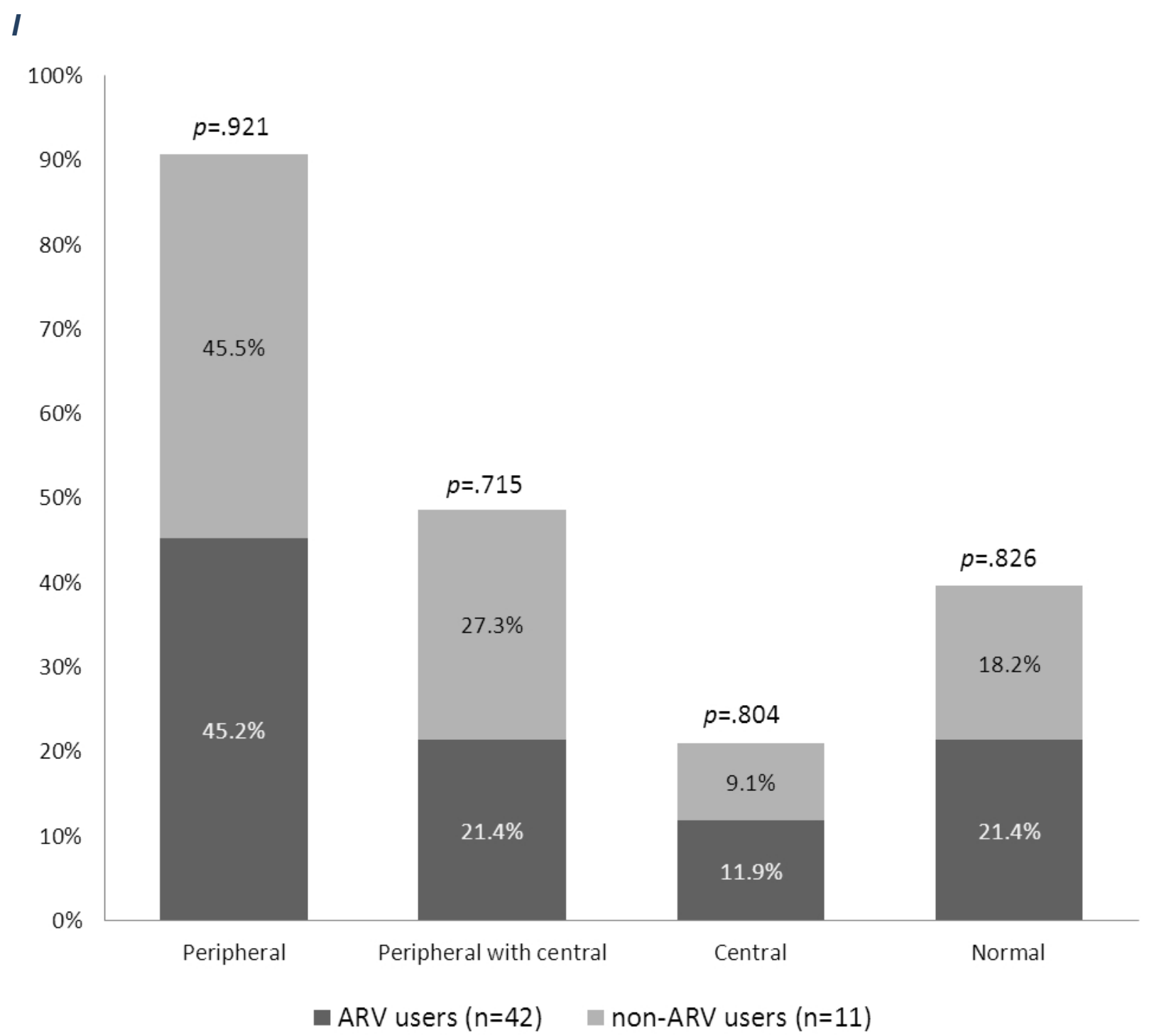

Fig. 3. Occurrence of vestibular involvement in HIV positive subjects with and without ARV therapies

\section{Discussion}

The current study demonstrated a significantly higher occurrence of vestibular involvement among adults with HIV than among those without HIV infections. There is a high likelihood of vestibular involvement in individuals with this disease, as demonstrated by the odds 
ratio. These findings correspond with previous studies that indicated a higher occurrence of vestibular dysfunction among adults with HIV [18,24-26,40]

Vestibular involvement presented throughout all categories of HIV disease progression. The occurrence of vestibular involvement increased from category 1 to category 2 , but not from category 2 to category 3. Peripheral vestibular involvement decreased from $18.9 \%$ in category 2 to $11.3 \%$ in category 3 . This was quite surprising, considering that three other studies showed a high occurrence of vestibular involvement in the advanced categories, particularly category $3[17,18,26]$. A possible suggestion for this equal occurrence in vestibular involvement in category $2(30.2 \%)$ and category $3(30.1 \%)$ and the decrease in peripheral vestibular involvement from category 2 to category 3 might be related to ARV therapies. In category 2, 38.2\% of subjects with HIV used ARV therapies, while $40.5 \%$ of subjects in category 3 used ARV therapies. Administering ARV therapies and highly active antiretroviral therapy (HAART) has shown to prevent the progression of HIV [41]. A recent study [42] demonstrated no association between peripheral vestibular impairment among HIV positive subjects using HAART and HIV negative subjects. In this regard Cohen and colleagues [42] suggested a therapeutic effect on immunity. ARV therapies aim to restore immune function and help protect the body against secondary opportunistic infections that are associated with vestibular dysfunction. This, in turncan reduce the occurrence of peripheral vestibular dysfunction in advanced stages of the disease. Another possible explanation for the decrease in peripheral vestibular involvement in the advanced category, where the majority of subjects $(n=13 / 18)$ were asymptomatic, might be that vestibular function was being compensated for centrally with increased disease duration. However, the subjects with HIV did not know when they became infected with the virus and therefore the relationship between disease duration, disease category and compensated vestibular function is unclear. With such a multifactorial disease, a repeated measures within subjects design would have provided more conclusive evidence.

This study, together with two recent studies [17,18], showed that signs of central vestibular involvement increased throughout disease progression. These findings are in agreement with other studies $[24,40,43]$ that demonstrated an association with centrally mediated ocular motor abnormalities, consistent with CNS dysfunction among HIV positive individuals. The occurrence of signs of central vestibular involvement in this study was 
smaller compared to those described by Teggi and colleagues [17,18]. A possible explanation may be that the sample of subjects in this study consisted of both symptomatic and asymptomatic individuals and that almost two-thirds (62.3\%) were asymptomatic. The studies by Teggi and colleagues only included symptomatic subjects, which could explain the higher occurrence of vestibular dysfunction, particularly central vestibular dysfunction, in their studies.

A higher occurrence of peripheral vestibular involvement compared to central vestibular involvement was evident in the current sample of HIV positive adult subjects. The occurrence of peripheral vestibular involvement in adult subjects with HIV may be attributed to related opportunistic infections and malignancies. Opportunistic bacterial and viral infections have been implicated to involve the sensory structures of the inner as well as the vestibulocochlear nerve (NVIII). Commonly occurring diseases that result in dysfunction of the vestibular end-organs and vestibular portion of NVIII are, among others, vestibular neuritis and labyrinthitis, otosyphilis Ramsay Hunt syndrome and herpes simplex virus [11,12,22,44-47]. According to Arbusow and colleagues [48] the mechanisms of peripheral vestibular involvement caused by opportunistic infections such as the herpes simplex virus involves a migration of the virus from the vestibular ganglia to the labyrinth of the vestibular end-organ, which may eventually result in vestibular deafferentation. However, these diagnoses of opportunistic infections were not documented in any of the subjects' medical records when data was collected.

The significantly higher occurrence of peripheral vestibular involvement found in the subjects with HIV compared to those without HIV, could indicate that HIV affects the peripheral vestibular system directly. Abnormalities of the cVEMP suggested involvement of the saccule (otolith organ) and inferior vestibular nerve, while test procedures such as the head impulse, head shake, positional and caloric tests suggested involvement of the semi-circular canals and superior vestibular nerve. Several reports have indicated that HIV may directly affect the auditory system $[49,50]$ and because of the close anatomical proximity, shared nerve innervations and blood supply this may also affect the peripheral vestibular system. Mathews and colleagues [51] recently showed that HIV affects the auditory-vestibular pathway as demonstrated by the high incidence of sensorineural hearing loss and associated vestibular dysfunction in HIV positive compared to HIV 
negative subjects. However, the nature of this association in individuals with HIV is not yet clear. A post-mortem study [52] suggested that not only is the CNS vulnerable to direct viral infections, but the peripheral vestibular labyrinth neuroepithelium displayed a presence of viral-like particles that seemed characteristic of the human immune-deficiency virus. Specifically, pathologies were observed within the semi-circular canals as well as in both the otolith organs. Another report [53] indicated similar results of abnormalities within the otolith organs, together with precipitations in the fluid filled semi-circular canals.

Almost two thirds of the subjects with HIVIAIDS who presented with vestibular involvement did not report any vestibular symptoms since contracting HIV. Previous reports [24,26] also indicated some vestibular involvement, even in asymptomatic individuals with no neurological or vestibular symptoms. The results of the current study indicated that vestibular involvement, particularly peripheral vestibular involvement, occurred among asymptomatic subjects with HIV. A possible explanation for observing vestibular involvement in asymptomatic HIV positive subjects might be the fact that cVEMP was included, making the test battery more sensitive for identifying peripheral vestibular disorders. Abnormal cVEMP occurred in 35.8\% $(n=19)$ of the subjects with HIV, of which $15.1 \%(n=8)$ had only an abnormal cVEMP with no other signs of peripheral vestibular involvement. Including the cVEMP was the first of its kind in a study of this nature, and was able to detect involvement of not only the saccule in the vestibular end-organ, but also of the functioning and integrity of the inferior branch of the vestibular nerve (NVIII). Nola and colleagues [54] showed the diagnostic value of the cVEMP in subjects with vestibular neuritis. They demonstrated abnormal caloric responses with normal cVEMP responses in subjects with superior vestibular neuritis, and normal caloric responses with abnormal cVEMPs in subjects with inferior vestibular neuritis. Some subjects with abnormal cVEMPs reported rotatory vertigo while others reported disequilibrium. This data, together with findings of Aw and colleagues [55] indicate that a virus can not only affect the superior vestibular nerve, but also the inferior vestibular nerve. Eighth cranial nerve neuropathies have been identified through evoked potentials such as auditory brainstem responses (ABR) among individuals with HIV, since ABR adequately sensitive to detect early subclinical and clinical neural auditory pathologies [19,56,57]. cVEMP testing might therefore be considered useful in identifying peripheral vestibular involvement in individuals with HIV. Hausler and colleagues [26] found only central vestibular involvement 
in their sample of asymptomatic subjects, while peripheral vestibular involvement was reported only in the symptomatic subjects during the advanced stages of disease progression. In a study by Castello and colleagues [24] HIV infection seemed to have no effect on the vestibular labyrinth or eighth cranial nerve in the sample of asymptomatic subjects.

No other studies compared the occurrence of vestibular involvement in symptomatic and asymptomatic HIV positive subjects. The current findings therefore suggest that vestibular involvement, particularly peripheral vestibular involvement in HIVIAIDS, may be present, but sub-clinical in some patients Primary health care providers should be aware that patients with HIV have an increased prevalence of vestibular dysfunction. Once patients have symptoms, primary health care providers could consider referring them for vestibular assessments and vestibular rehabilitation therapy. Preventative measures could be taken to reduce the risk of serious, painful falls that can impede quality of life and activities of daily living. This may be especially important in light of recent evidence of a significant link between vestibular dysfunction and the risk of falling, even in asymptomatic subjects [58].

The occurrence of vestibular involvement was equal in subjects receiving ARV therapies compared to those not receiving ARV therapies. This lack of differences was a surprising finding, considering a recent report [42] that demonstrated no significant difference in the prevalence of vestibular abnormalities in subjects with HIV using HAART and subjects without HIV. We therefore expected less vestibular abnormalities in HIV positive subjects using ARV therapies. The findings indicated that the administration of ARV therapies does not reduce the risk of having vestibular abnormalities. A possible explanation for dissimilar results of this study and that of Cohen and colleagues [42], may be attributed to the vestibular test procedures employed. The current study employed a test battery consisting of various tests of peripheral and central vestibular function. It included objective measurements, such as caloric tests and cVEMP recordings. This might have made the study more sensitive for identifying vestibular disorders, even sub-clinical ones. The occurrence of vestibular test abnormalities despite the administration of ARV therapies, might be attributed to possible vestibulo-toxic properties. A detailed summary of reports on ototoxicity related to ARV can be found elsewhere [59] and some of the therapies included in this summary are, among others, Zidovudine (AZT), Didanosine (ddl), Stavudine (d4T), 
Lamivudine (3TC) and Nevirapine (NVP). The majority of these studies demonstrated that NRTIs were the most likely cause of the subjects' hearing loss. A recent study [60] indicated that their sample of HIV positive subjects who received ARV therapies showed more abnormalities in their auditory evoked potentials, such as auditory brainstem response, than those who did not receive ARV therapies. They suggested that the auditory nerve and brainstem structures were at-risk due to the ototoxic effect of these agents. This is a complex interaction, because it seems to result in ototoxicity for specific cases with specific ARV combinations, even involving interactions with other drugs, alongside possible person-specific susceptibility factors [59]. Reports on ototoxic ARV agents may suggest this as a possibility to explain the occurrence of vestibular involvement in HIV in earlier stages of the disease. ARV therapies improve general immunity avoiding secondary infections, therefore the improvement in the advanced stages may not just be due to ARV therapies but may include to central compensation. .

\section{Conclusion}

There is a high occurrence of vestibular involvement in adults with HIV. Primary health care providers could screen HIV positive patients to ascertain if there are symptoms of vestibular involvement. If there are any, then they may consider further vestibular assessments and subsequent vestibular rehabilitation therapy. HIV positive subjects have a high risk for vestibular abnormalities; these may occur despite being asymptomatic and/or receiving ARV therapies.

\section{Conflict of interest}

The authors declare that they have no conflict of interest.

\section{References}

[1] UNAIDS. UNAIDS report on the global AIDS epidemic. 2010; Available at:

http://www.unaids.org/globalreport/Global report.htm. Accessed December 7, 2010.

[2] Heinze BM, Swanepoel DW, Hofmeyr LM. Systematic review of vestibular disorders related to human immunodeficiency virus and acquired immunodeficiency syndrome. J Laryngol Otol 2011;125(9):881-890. 
[3] Fan HY, Conner RF, Villarreal LP. AIDS science and society. Fourth ed. Sudbury, Massachusetts: Jones and Bartlett Publishers; 2004.

[4] Evian C. Primary AIDS care. Third ed. Houghton: Jacana Education; 2000.

[5] Barzan L, Tavio M, Tirelli U, Comoretto R. Head and neck manifestations during HIV infection. J Laryngol Otol 1993 02;107(2):133-136.

[6] Lubbe DE. HIV and ENT. CME: Your SA journal of CPD 2004:250-253.

[7] Marsot-Dupuch K, Quillard J, Meyohas MC. Head and neck lesions in the immunocompromised host. Eur Radiol Suppl 2004 03/01;14(3):E155-E167.

[8] Salzer TA. Neurotologic manifestations of HIV infection. 1994; Available at: http//:www.bcm.edu/oto/grand/32494.html. Accessed June 22, 2009.

[9] Somefun A, Nwawolo CC, Okeowo PA, Ogban LU, Akanmu AS, Okanny CC, et al. Otorhinolaryngological manifestations of HIVIAIDS in Lagos. Niger Postgrad Med J 2001 01/01;8(4):170-174.

[10] Cohen BA, Berger JR. Other opportunistic infections of the central nervous system in AIDS. Handb Clin Neurol 2007;85:185-219.

[11] Gurney TA, Murr AH. Otolaryngologic manifestations of human immunodeficiency virus infection. Otolaryngol Clin North Am 2003;36(4):607-624.

[12] Moayedi S. Head, Neck and Ophthalmologic Manifestations of HIV in the Emergency Department. Emerg Med Clin North Am 2010;28(2):265-271.

[13] Chan SY, Medhi M, Ekbote A, Moses S, Sibtain N, Andrews T, et al. Syphilis causing hearing loss. Int J STD AIDS 2008;19(10):721-722.

[14] Devaleenal DB, Ahilasamy N, Solomon S, Kumarasamy N. Ramsay hunt syndrome in a person with HIV disease. Indian J Otolaryngol 2008;60(2):171-173.

[15] Matas CG, Sansone AP, lorio MCM, Succi RCM. Audiological evaluation in children born to HIV positive mothers. Rev Bras Otorinolaringol 2000;66(4):317-324.

[16] Palacios GC, Montalvo MS, Fraire MI, Leon E, Alvarez MT, Solorzano F. Audiologic and vestibular findings in a sample of Human Immunodeficiency Virus type-1-infected Mexican children under Highly Active Antiretroviral Therapy. Int J Pediatr Otorhinolaryngol 2008;72(11):1671-1681.

[17] Teggi R, Giordano L, Pistorio V, Bussi M. Vestibular function in HIV patients:

preliminary report. Acta Otolaryngol 2006; Italy(26):140-146.

[18] Teggi R, Ceserani N, Luce FL, Lazzarin A, Bussi M. Otoneurological findings in human immunodeficiency virus positive patients. J Laryngol Otol 2008;122(12):1289-1294. 
[19] Vincenti V, Pasanisi E, Bacciu A, Giordano D, Di Lella F, Guida M, et al. Cochlear implantation in a human immunodeficiency virus-infected patient. Laryngoscope 2005;115(6):1079-1081.

[20] Chandrasekhar SS, Connelly PE, Brahmbhatt SS, Shah CS, Kloser PC, Baredes S. Otologic and audiologic evaluation of human immunodeficiency virus- infected patients. Am J Otolaryngol 2000;21(1):1-9.

[21] Lalwani AK, Sooy CD. Otologic and neurotologic manifestations of acquired immunodeficiency syndrome. Otolaryngol Clin North Am 1992;25(6):1183-1198.

[22] Prasad HKC, Bhojwani KM, Shenoy V, Prasad SC. HIV manifestations in otolaryngology. Am J Otolaryngol 2006;27(3):179-185.

[23] Zuniga J. Communication disorders and HIV disease. J Int Assoc Physicians AIDS Care 1999;5(4):16-23.

[24] Castello E, Baroni N, Pallestrini E. Neurotological and auditory brain stem response findings in human immunodeficiency virus-positive patients without neurologic manifestations. Ann Otol Rhinol Laryngol 1998;107(12):1054-1060.

[25] Dellepiane M, Medicina MC, Mora R, Salami A. Static and dynamic posturography in patients with asymptomatic HIV-1 infection and AIDS. Acta Otorhinolaryngol Ital 2005;25(6):353-358.

[26] Hausler R, Vibert D, Koralnik IJ, Hirschel B. Neuro-otological manifestations in different stages of HIV infection. Acta Otolaryngol Suppl 1991(481):515-521.

[27] Maes L, Dhooge I, D'haenens W, Bockstael A, Keppler H, Philips B, et al. The effect of age on the sinusoidal harmonic acceleration test, pseudorandom rotation test, velocity step test, caloric test, and vestibular evoked myogenic potential test. Ear Hear 2010;31:8494.

[28] Dorrington R, Bourne D. Has HIV prevalence peaked in South Africa? - Can the report on the latest antenatal survey be trusted to answer this question? S Afr Med J 2008;98(10):754-755.

[29] Goebel JA. Practical management of the dizzy patient. Second ed. Philadelphia, USA: Lippincott Williams \& Wilkins; 2008.

[30] Jerger J. Clinical experience with impedance audiometry. Arch Otolaryngol 1970;92:311-324. 
[31] Wester M. Vestibular evoked myogenic potentials. In: Atcherson SR, Stoody TM, editors. Auditory electrophysiology: A clinical guide New York, NY: Thieme Medical Publishers; 2012. p. 289-306.

[32] Colebatch JG, Halmagyi GM, Skuse NF. Myogenic potentials generated by a clickevoked vestibulocollic reflex. J Neurol Neurosurg Psychiatry 1994;57(2):190-197. [33] Vanspauwen R, Wuyts FL, Van De Heyning PH. Improving vestibular evoked myogenic potential reliability by using a blood pressure manometer. Laryngoscope 2006;116(1):131-135.

[34] Maes L, Vinck BM, De Vel E, D'haenens W, Bockstael A, Keppler H, et al. The vestibular evoked myogenic potential: A test-retest reliability study. Clin Neurophysiol 2009;120(3):594-600.

[35] Akin FW, Murnane OD. Vestibular evoked myogenic potentials. In: Jacobson GP, Shepard NT, editors. Balance function assessment and management San Diego; Oxford; Brisbane: Plural Publishing; 2008. p. 405-434.

[36] Zwergal A, Rettinger N, Frenzel C, Dieterich M, Brandt T, Strupp M. A bucket of static vestibular function. Neurology 2009;72(19):1689-1692.

[37] Kerber KA, Ishiyama GP, Baloh RW. A longitudinal study of oculomotor function in normal older people. Neurobiol Aging 2006;27(9):1346-1353.

[38] Baloh RW, Enrietto J, Jacobson KM, Lin A. Age-related changes in vestibular function a longitudinal study. Ann New York Acad Sci 2001;942:210-219.

[39] Jongkees LB, Maas J, Philipszoon A. Clinical nystagmography: a detailed study of electro-nystagmography in 341 patients with vertigo. Pract Otorhinolaryngol (Basel) 1962;24(1):65-93.

[40] Johnston JL, Miller JD, Nath A. Ocular motor dysfunction in HIV-1-infected subjects: A quantitative oculographic analysis. Neurology 1996;46(2):451-457.

[41] Schneider MF, Gange SJ, Williams CM, Anastos, K., Greenblatt, R.M., Kingsley L, Detels R, et al. Patterns of the hazard of death after AIDS through the evolution of antiretroviral therapy. AIDS 2005;19(17):2009-2018.

[42] Cohen HS, Cox C, Springer G, Hoffman HJ, Young MA, Margolick JB, et al. Prevalence of abnormalities in vestibular function and balance among HIV-seropositive and HIV-seronegative women and men. PLoS ONE 2012;7(5). 
[43] Sacktor N, Lyles RH, Skolasky R, Kleeberger C, Selnes OA, Miller EN, et al. HIVassociated neurologic disease incidence changes: Multicenter AIDS Cohort Study, 19901998. Neurology 2001;56(2):257-260.

[44] Goldani LZ, Ferreira Da Silva LF, Dora JM. Ramsay Hunt syndrome in patients infected with human immunodeficiency virus. Clin Exp Dermatol 2009;34(8):e552-e554.

[45] Macher AM. Otosyphilis: auditory and vestibular presentations. American Jails 2008;March/April.

[46] Pérez-Larraya JG, Riverol M. Facial diplegia and vestibular neuritis secondary to HIV seroconversion syndrome. Can J Neurol Sci 2009;36(4):527-528.

[47] Jae JS, Lee H-, Sung WC, Soon JH. Bilateral otosyphilis in a patient with HIV infection. Eur Arch Otorhinolaryngol 2005;262(12):972-974.

[48] Arbusow V, Theil D, Strupp M, Mascolo A, Brandt T. HSV-1 not only in human vestibular ganglia but also in the vestibular labyrinth. Audiol Neurootol 2001;6(5):259-262. [49] Rey D, L'Héritier A, Lang JM. Severe ototoxicity in a health care worker who received postexposure prophylaxis with stavudine, lamivudine, and nevirapine after occupational exposure to HIV [2]. Clin Infect Dis 2002;34(3):418-419.

[50] Rinaldo A, Brandwein MS, Devaney KO, Ferlito A. AIDS-related otological lesions. Acta Otolaryngol 2003;123(6):672-674.

[51] Mathews SS, Albert RR, Job A. Audio-vestibular function in human immunodeficiency virus infected patients in India. Indian journal of sexually transmitted diseases 2012;33(2):98-101.

[52] Pappas Jr. DG, Roland Jr. JT, Lim J, Lai A, Hillman DE. Ultrastructural findings in the vestibular end-organs of AIDS cases. Am J Otol 1995;16(2):140-145.

[53] Chandrasekhar SS, Siverls V, Chandra Sekhar HK. Histopathologic and ultrastructural changes in the temporal bones of HIV-infected human adults. Am J Otol 1992;13(3):207214.

[54] Nola G, Guastini L, Crippa B, Deiana M, Mora R, Ralli G. Vestibular evoked myogenic potential in vestibular neuritis. European Archives of Oto-Rhino-Laryngology 2011;268(11):1671-1677.

[55] Aw ST, Fetter M, Cremer PD, Karlberg M, Halmagyi GM. Individual semicircular canal function in superior and inferior vestibular neuritis. Neurology 2001;57(5):768-774. [56] Larsen CR. HIV-1 and communication disorders: What speech and hearing professionals need to know. San Diego; London: Singular Publishing Group; 1998. 
[57] Moazzez AH, Alvi A. Head and neck manifestations of AIDS in adults. Am Fam Physician 1998;57(8):1813-1822.

[58] Agrawal Y, Carey JP, Della Santina CC, Schubert MC, Minor LB. Disorders of balance and vestibular function in US adults: Data from the National Health and Nutrition Examination Survey, 2001-2004. Arch Intern Med 2009;169(10):938-944.

[59] Stearn N, Swanepoel DW. Sensory and neural auditory disorders associated with HIVIAIDS. In: Swanepoel DW, Louw B, editors. HIVIAIDS related communication, hearing and swallowing disorders San Diego, Oxford, Brisbane: Plural Publishing; 2010. p. 243288.

[60] Matas CG, Silva SM, de Almeida Marcon B, Gonçalves IC. Electrophysiological manifestations in adults with HIVIAIDS submitted and not submitted to antiretroviral therapy. Pro-Fono 2010;22(2):107-112. 